\title{
Nanoscale Molecular Quantification of Stem Cell-Hydrogel Interactions
}

Stephanie A. Maynard ${ }^{1 \Delta}$, Amy Gelmi ${ }^{1 \ddagger \Delta}$, Stacey C. Skaalure ${ }^{1}$, Isaac J. Pence ${ }^{1}$, Charlotte LeeReeves $^{1}$, Julia E. Sero ${ }^{1+\star}$, Thomas E. Whittaker ${ }^{1}$, Molly M. Stevens ${ }^{1 *}$

${ }^{1}$ Department of Materials, Department of Bioengineering and Institute of Biomedical Engineering, Imperial College London, London, SW7 2AZ, United Kingdom

$¥$ Current address: Applied Chemistry and Environmental Science, School of Science, RMIT University, Melbourne, VIC 3001, Australia

tt Current address: University of Bath, Claverton Down, Bath, BA2 7AY, United Kingdom $\Delta$ These authors contributed equally

*Corresponding author

\section{Supplementary Information}
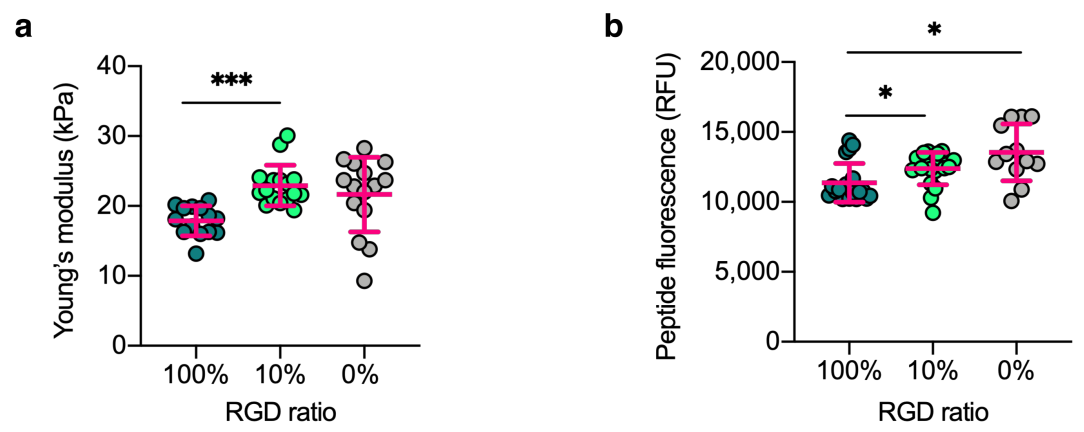

Figure S1. Characterization of RGD peptide-functionalized hydrogels. (a) Young's modulus of hydrogels was measured using AFM. $\mathrm{N}=14-16$ gels for each condition. Mean $\pm \mathrm{SD}$ for each condition are $18 \pm 2 \mathrm{kPa}, 23 \pm 3 \mathrm{kPa}$, and $22 \pm 5 \mathrm{kPa}$, for $100 \%, 10 \%$, and $0 \% \mathrm{RGD}$, respectively. (b) Fluoraldehyde assay of total peptide content in different hydrogel conditions. $\mathrm{N}=4$ measurements each from $4-5$ gels per condition. Brown-Forsythe and Welch unequal variances unpaired one-way ANOVA, Dunnett's T3 multiple comparison test. Dot plots represent mean $\pm \mathrm{SD} .{ }^{*} \mathrm{p}<0.05,{ }^{*} \mathrm{p}<0.01,{ }^{* * *} \mathrm{p}<0.001$. 


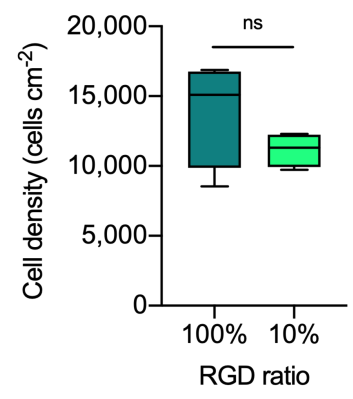

Figure S2. Analysis of number of cells bound to hydrogels. Total number of nuclei per $\mathrm{cm}^{2}$, as measured from images taken at 10x magnification within a $1.2 \times 1.2 \mathrm{~mm}$ region of hydrogel. $\mathrm{N}=4$ images per condition. Parametric unpaired two-tailed t-test. Box plots represent median $\pm I Q R$, whiskers represent minimum and maximum. ns $=$ not significant.

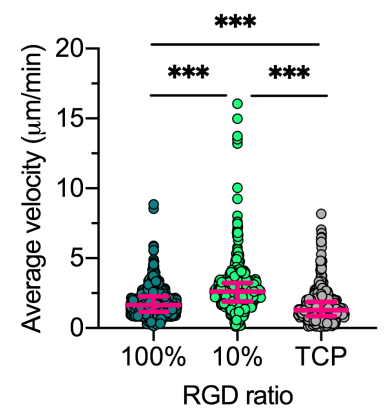

Figure S3. Average migration velocity of hMSCs. Average velocity of hMSC tracks on hydrogels of $100 \%$ and $10 \%$ RGD concentration, compared to TCP controls. $\mathrm{N}=613-1203$ tracks per condition. Nonparametric Kruskal-Wallis ANOVA with Dunn's multiple comparison test. Dot plots represent median \pm IQR. ${ }^{* * *} \mathrm{p}<0.001$. 

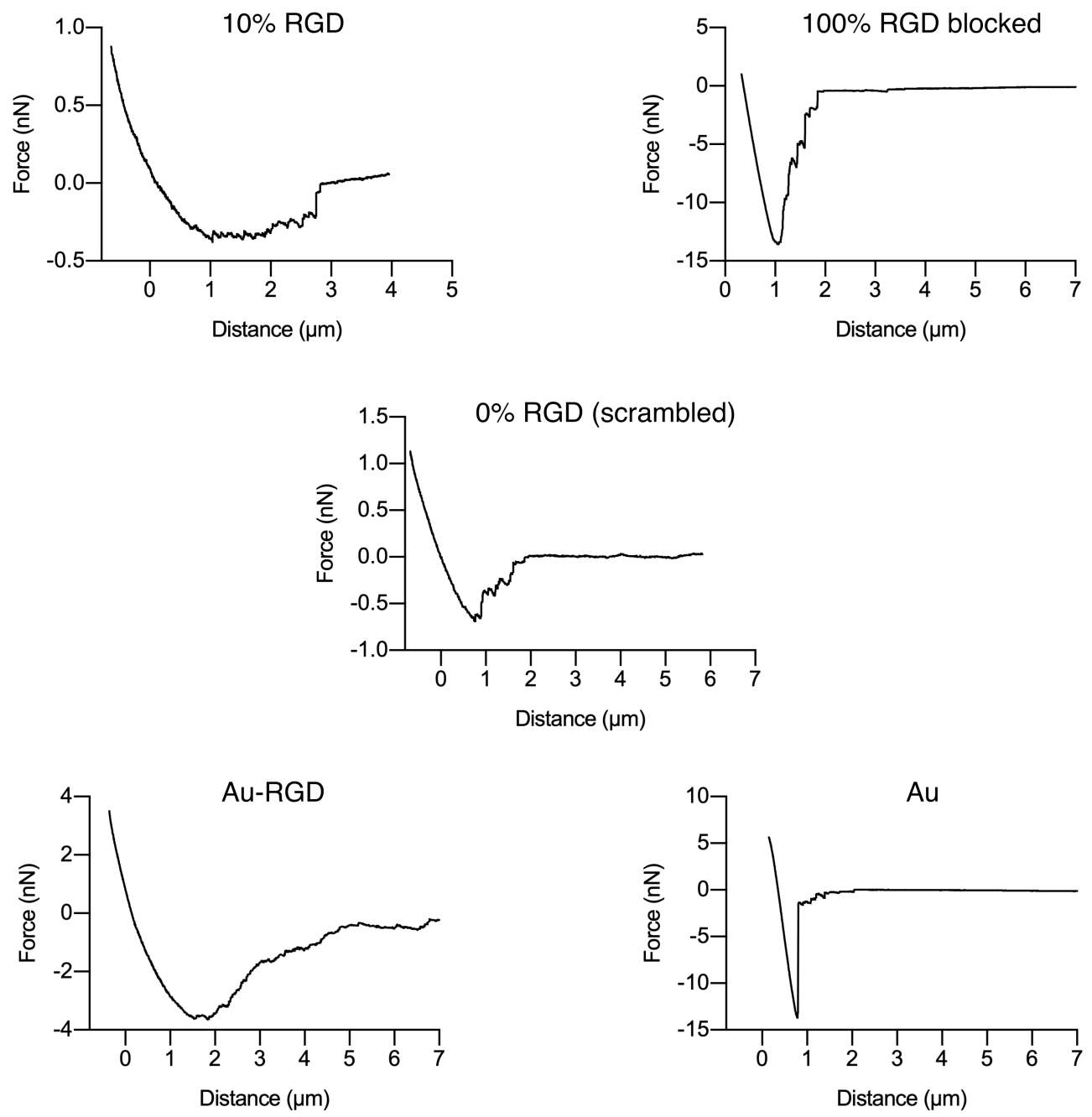

Figure S4. AFM force curve controls. Example force-distance curves of hMSC unbinding from 10\% RGD hydrogel, 100\% RGD hydrogel following blocking of the hMSC in RGD, 0\% RGD (scrambled) hydrogel, RGD functionalized gold surface (Au-RGD), and unfunctionalized gold surface (Au). 


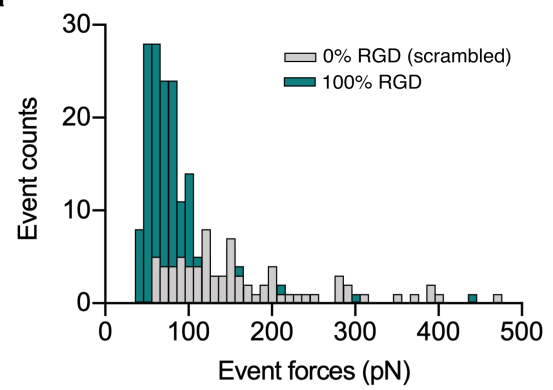

b

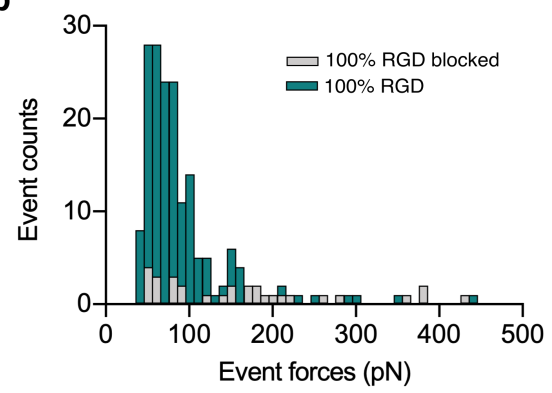

C

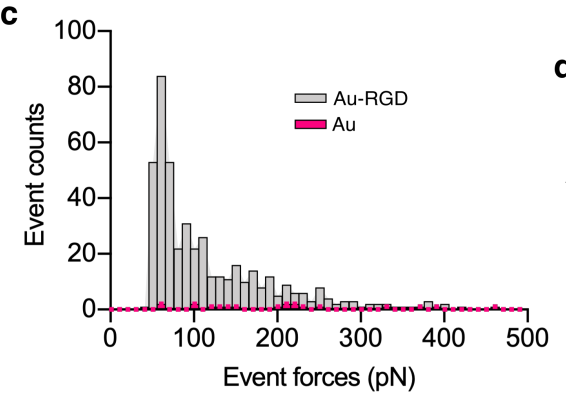

d

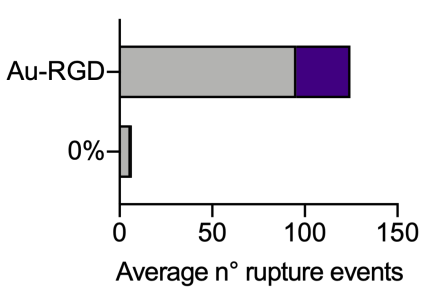

/ force displacement e

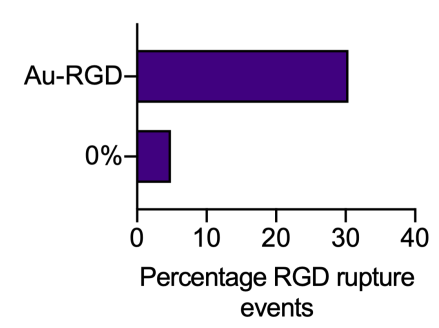

Figure S5. AFM event forces for controls. Comparison of total event forces when hMSCs were interfaced with (a) 100\% RGD hydrogel and 0\% RGD (scrambled) hydrogel, (b) 100\% RGD hydrogel and 100\% RGD hydrogel following RGD blocking of hMSC, (c) Au and RGD-functionalized Au. (d) Average total and RGD-specific rupture events on 0\% RGD hydrogel and RGD functionalized Au substrates. (e) Percentage of RGD rupture events per force displacement curve on 0\% RGD hydrogel and RGD functionalized Au substrates. $\mathrm{N}=3, \mathrm{n}=5$ per condition.
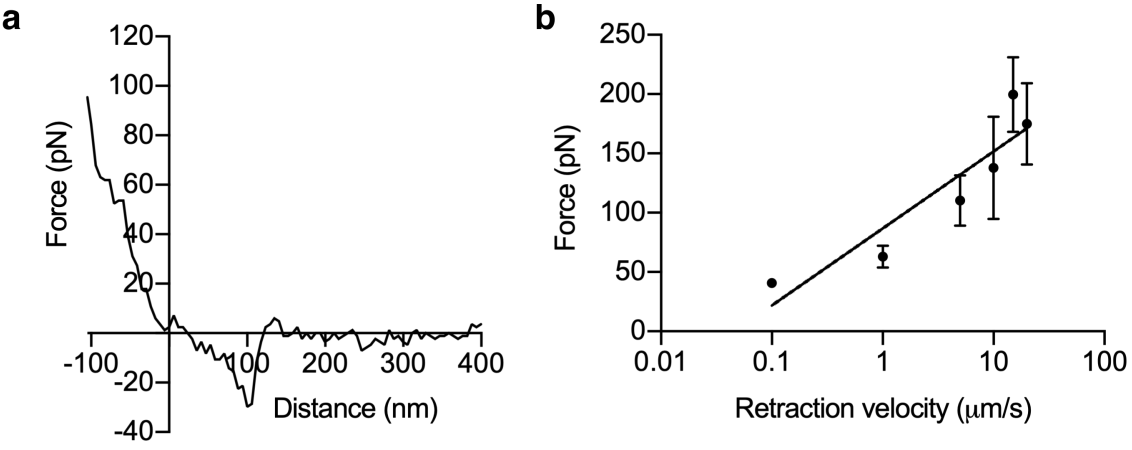

Figure S6. AFM loading rate. (a) Representative force-distance curve showing a single molecule unbinding event, between hMSC and 100\% RGD hydrogel with a retraction velocity of $0.1 \mu \mathrm{m} / \mathrm{s}$. (b) Dependence of the average rupture force measured for single molecule unbinding events on the $100 \%$ RGD hydrogel with loading rate. $\mathrm{N}=3, \mathrm{n}=40$ per retraction velocity condition. 
a

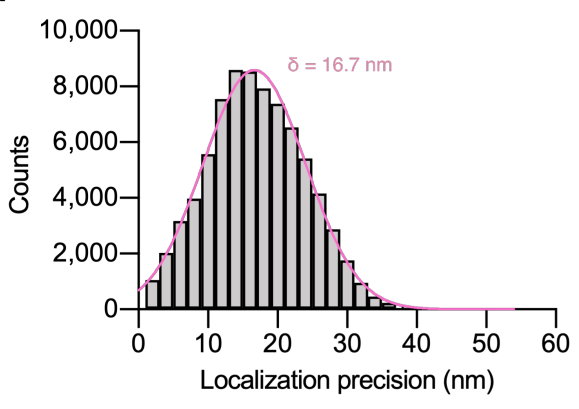

b

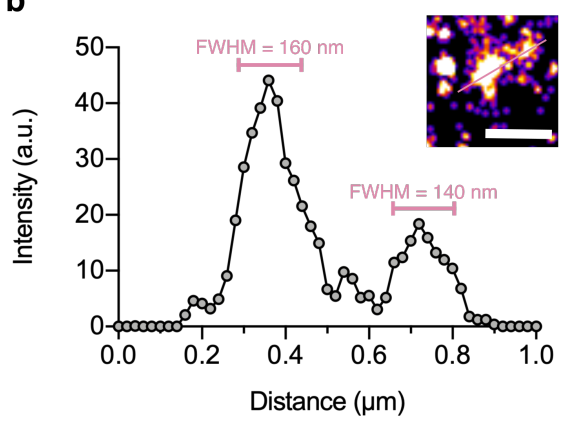

Figure S7. dSTORM localization precision. (a) Localization precision of single AlexaFluor647 detections was measured, median $=16.7 \mathrm{~nm}$. (b) Intensity profile of two clusters (line profile inset) gives a full width half maximum (FWHM) of 140-160 nm. Scale bar $=500 \mathrm{~nm}$.
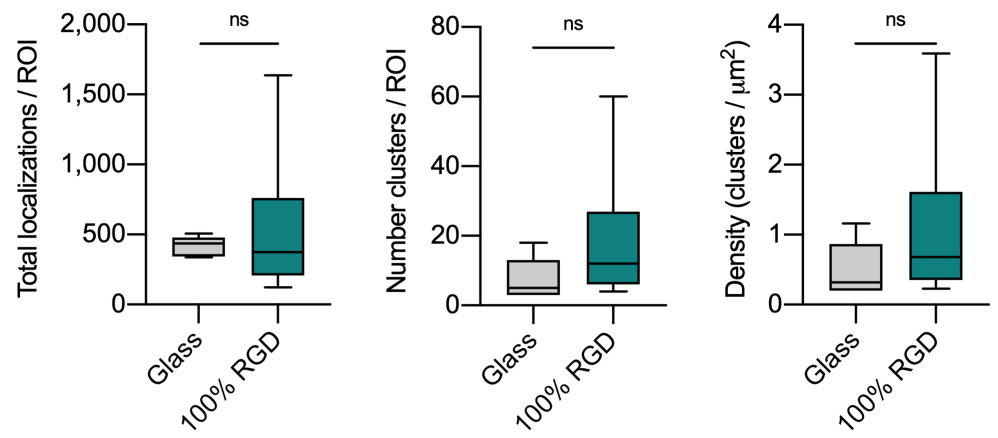

Figure S8. dSTORM cluster analysis for control. Analysis of total number of surface localizations, number of clusters and density of clusters of integrin $\alpha 5 \beta 1$ per ROI for hMSCs in contact with untreated glass control and 100\% RGD hydrogels. $\mathrm{N}=5-15$. Welch's unequal variances unpaired two-tailed t-test. Box plots represent median $\pm \mathrm{IQR}$. ns $=$ not significant. 